\title{
Sistema de costos por actividades en la industria del calzado
}

\author{
Amerio, María Anna \\ Viloria, Orlando \\ De Freitas, Vidalina \\ Nava, Luis*
}

\section{Resumen}

El presente trabajo resalta la importancia del sistema de costos basado en actividades y su adaptación al sector de la pequeña y mediana industria del calzado. El objetivo fundamental es diseñar un sistema de asignación de costos basado en actividades para el sector mencionado. Para ello se realizó una revisión documental, observación directa de los procesos productivos y se aplicaron entrevistas a los trabajadores involucrados en la producción. Se concluye que el sistema de costos debe adaptarse a la estructura de costos y a los procesos productivos del sector calzado. De esa manera, el departamento de contabilidad suministrará a los gerentes, el costo del proceso de producción, de las actividades que lo conforman y de los productos, enfocada a la gestión por actividades $y$ al mejoramiento continuo.

Palabras clave: Costos, gerencia, finanzas, sistemas contables, producción.

\section{Recibido: 98-06-05 . Aceptado: 98-11-05}

- Apoyo Financiero: Universidad Simón Bolivar.

Los autores son Profesores de la Universidad Simón Bolivar, Sede del Litoral. Lic. Contaduría Pública, Msc. Finanzas; Lic. Computación, Msc. Ingenieria de Sistemas; Ing. en Computación, Msc en Ingeniería de Sistemas; y Economista, Msc. Relaciones Económicas Internacionales, respectivamente. Dirección: Valle de Camuri Grande, Edo. Vargas; Telf. (031)722911 al 16 ext. $252 / 129$ y $02-627054$. 


\section{Activity based costing in Shoe Industry}

\section{Abstract}

This paper points out the importance of activity based costing (ABC) and its adaption to the small and medium sized shoe industry sector. The basic objective is to design a system of cost assignments based on activities in the sector mentioned. In order to do this, the revision of documents was undertaken, as well as direct observation of the productive process and the application of a survey questionaire to production workers. The conclusion was that the cost system should adapt itself to the cost structure and the productive process of the shoe industry. In this way, the accounting department will present management with information about the costs of production, the costs of each activity in the productive process as well as the total cost, focusing on management by activity and continual improvement of the process.

Key words: Costs, management, finance, accounting systems, production.

\section{Introducción}

En las empresas exitosas, para alcanzar y mantener la competitividad en el ámbito internacional, los gerentes deben tomar decisiones inteligentes y oportunas en relación con los procesos de planeación, organización y control de las operaciones, tanto a corto como a largo plazo.

En ese sentido Burgos, (1994: 96) afirma que la toma de decisiones es:

\footnotetext{
"...La columna vertebral de la gerencia y los niveles de excelencia en la actuación de un gerente no provienen sino de razonables prácticas gerenciales basadas en la tecnología gerencial y experiencia, a través de una toma de decisiones resultante de una mezcla apropiada de racionalidad e intuición."
}

En el proceso de toma de decisiones, la información de costos se conside- ra una herramienta fundamental y su análisis ayuda a la gerencia a tomar decisiones acertadas, orientadas a que su organización penetre y se mantenga en el ambiente competitivo actual.

Al respecto, Shank y Govindarajan (1996) considera que el análisis de costos es el proceso para estimar el impacto financiero que pueden ejercer las decisiones gerenciales; por lo tanto, los datos de costos se utilizan para desarrollar estrategias superiores $y$, un conocimiento sofisticado de la estructura de costos es de gran ayuda en la búsqueda de ventajas competitivas sostenibles.

Por su parte, FIM Productividad (1992), destaca la necesidad de utilizar sistemas de contabilidad de costos y velar por su vigencia e idoneidad, de manera de realimentar oportunamente las es. trategias de mejoramiento de la productividad y calidad. 
En el caso venezolano, las investigaciones realizadas por Kaplan (1994), Enright et al (1994) y Linares (1996), concluyen que las pequeñas y medianas empresas (Pymi) no procesan datos de costos de manera organizada a través de un sistema contable. En consecuencia, los gerentes no disponen de información de costos relevante, práctica y confiable para estudiar cada alternativa de decisión.

De la situación planteada se deduce la importancia de implantar sistemas de costos en las Pymi venezolanas, que proporcionen reportes de costos y satisfagan los requerimientos de información de los gerentes.

En ese sentido, el sistema de contabilidad de costos debe ser compatible con la naturaleza y el tipo de operaciones que realiza una empresa manufacturera. Para ello es importante que en su diseño se consideren las caracteristicas especificas de cada sector industrial.

Por otro lado, Amerio et al (1997) presentan a la pequeña y mediana industria del calzado venezolana como un ejemplo de la situación planteada, así como la necesidad de elaborar un sistema de contabilidad de costos adaptado al sector con el fin de suministrar información de costos acorde con las teorías modernas y utilizadas por los gerentes a escala internacional.

El presente trabajo se orienta al diseño de un sistema de asignación de costos basado en actividades para el sector calzado, que suministre la información que requieren los gerentes actualmente para apoyar sus decisiones.

Para desarrollar el diseño se establecieron las bases conceptuales mediante el análisis de las teorias de costos, tanto en el área de contabilidad como en el área gerencial. Posteriormente se estudiaron los procesos productivos del calzado, recolectando información a través de la observación directa y las entrevistas aplicadas a los gerentes de las empresas del sector y a representantes de la Cámara Venezolana de la Industria del Calzado.

El sistema de asignación de costos obtenido, servirá como base a los sistemas de presupuestos y sistemas de contabilidad de costos en las pequeñas y medianas industrias del sector calzado, sustentado en el desarrollo y aplicación de las teorías que predominan en las empresas competitivas a nivel internacional, recomendadas por Kaplan (1994), Lorino (1993) y Horngren, Foster, y Srikant (1996).

\section{Sistema de asignación de costos basado en actividades (ABC)}

Los expertos como Andersen (1996), Baker (1994), Kaplan (1994) y Acosta (1996), definen al sistema de costos basado en actividades (ABC) como una técnica en donde el modelo de costos se orienta a las actividades necesarias para producir, comercializar y distribuir productos y servicios.

Howell y Soucy (1991) y Castillo (1995) agregan que las actividades se combinan en cadenas o en redes de actividades denominadas procesos, destinadas al logro de un objetivo global, es decir, a una salida global material o inmaterial. Por lo tanto, el costo de cada proceso incluye la sumatoria de las actividades que lo conforman. 
A través del sistema se alinean los costos de la organización con las actividades de operación y todas las actividades operativas son identificadas y asignadas a los productos. Se analizan los procesos para determinar los tipos de actividades; las actividades absorben los costos y son definidas y asociadas con productos utilizando el concepto de generadores de costos. En consecuencia, se consideran tanto los procesos como los productos.

\section{Ventajas del sistema de asignación de costos basado en actividades}

En sus investigaciones, Horngren, Foster, y Srikant (1996), afirma que un gran porcentaje de empresas en el ámbito internacional han sustituido el sistema de costos basado en las teorías tradicionales, por el sistema de costos basado en actividades ( $A B C$ ), debido a que proporciona información amplia y más exacta. Tal decisión se fundamenta en las ventajas que se mencionan a continuación:

1. El objetivo primordial es el rastreo preciso de los costos de las actividades y la asignación de éstos a los productos.

2. Se orienta a los procesos y se proyecta sobre todas las actividades de la empresa. Es decir, proporciona una visión global del proceso.

3. Los métodos de asignación utilizados reparten equitativamente los costos a los productos o servicios, considerando que los costos consumidos por un producto no varian en proporción a la distribución básica asignada para los volumenes producidos.
4. Se utiliza un mayor número de bases de asignación, representando factores de costos o variables no financieras.

5. Se mantiene una relación de causa efecto. El sistema considera que son las actividades las que generan los costos adicionales de un producto, por lo que permite enfocar la atención hacia la causa de los costos. En tal sentido, los objetos de costos son evaluados sobre la base de las actividades.

6. Proporciona información de costos por actividad, por proceso, por unidad de producto o servicio u otro objetivo de costos; suministra costos confiables de los productos debido a que permite un alto grado de precisión en la asignación de costos a los mismos; informa sobre las actividades fundamentales y los generadores de costos; por último, permite mejor entendimiento de la estructura de costos.

La información mencionada facilita las decisiones estratégicas y permite determinar oportunidades para reducir los costos, lograr la identificación del valor agregado y no agregado de las actividades, medir el desempeño y alcanzar el mejoramiento de la calidad.

\section{Desarrollo de un sistema de costos basado en actividades}

De acuerdo con Lorino (1993), Polimeni, Fabozzi y Adelberg (1994) y Horngren, Foster, y Srikant (1996), el desarrollo de un sistema de costos $A B C$ presenta un alto nivel de complejidad en la definición de las actividades, en la selec- 
ción de los factores de costos y en la aceptación por parte de los empleados y gerentes involucrados.

Por lo tanto, para simplificar el proceso de implantación en el presente trabajo se aplicó una metodología, basada en Lorino (1993) y demás expertos, la cual se explica a continuación:

1. Definición de los procesos: En esta fase se identificaron los procesos $y$ las actividades que los conforman. Consistió en formular los objetivos estratégicos y operativos, crear un equipo de trabajo, elaborar un plan de recolección de datos, recopilar la información necesaria para identificar plenamente los procesos, y limpiarla, ordenarla y precisarla.

2. Definición de las actividades: consistió en establecer una estructura de actividades para el proceso de diseño y producción. Se analizaron los procesos desarrollados en cada función en la línea de producción, tomando una visión horizontal de todo el proceso productivo y de distribución del producto final. Por tal motivo, la selección y clasificación de las actividades se realizó bajo un esquema adaptable a cualquier empresa del sector, con las caracteristicas de flexibilidad necesarias. Además, se estableció un nivel de detalle adecuado de acuerdo con Lorino (1993), para evitar la complejidad por exceso de actividades.

3. Descripción de las actividades del proceso productivo, identificándolas con un nombre reconocible, expresado en el lenguaje de la empresa con un contenido breve y objetivo. Para ello se utilizaron fichas prácticas que permitieron agrupar y describir el detalle de cada actividad con el propósito de conformar una base de datos.

4. Elaboración del plan de cuentas: Con el fin de desarrollar el plan de cuentas para el registro de los costos estimados y reales se estudiaron los recursos que consumen las actividades del proceso respectivo, se conformaron grupos de cuentas por recursos (Materia Prima, Mano de Obra y Gastos de Fabricación) y por las actividades que conforman el proceso que origina el costo de producción.

5. Selección de las unidades de trabajo o de medida uniformes para la asignación de costos en el proceso productivo. Con el fin de calcular el costo unitario de cada actividad de producción se divide el costo total de la actividad por la unidad de trabajo escogida. La selección se basó en las unidades de trabajo recomendadas por Lorino (1993), Acosta (1996) y Castillo (1995), agrupándolas en cuatro categorias en función del tipo de gestión: a) Gestión de producción, utilizándo medidas de flujo el número de piezas, operaciones, productos, dias u horas. b) Gestión comercial, financiera, de compras y recursos humanos; considerando como medida las transacciones, tales como el número de recepciones, pedidos, facturas, órdenes de compra o clientes. c) Base de datos utilizando como unidades de trabajo el número de clientes, proveedores y caracteres técnicos de los productos. d) La última categoría comprende la contabilidad y los parámetros de gestión para medir el costo, es decir, los gastos, precios valores de activos y volúmenes. 
6. Determinación de los criterios de asignación de costos a las actividades y a las unidades producidas: En esta fase se establecieron las bases para medir el consumo de los recursos y su repartición entre las actividades que conforman el proceso de diseño y producción.

7. Diseño del sistema de asignación de costos basado en actividades: La mejor manera de conceptualizar la forma en que los datos se mueven a través de la organización, los procesos o transformaciones que sufren los datos y las salidas, es a través de los diagramas de flujo de datos, tal como lo señalan Kendall y Kendall (1997). Por lo tanto, se diseñaron los diagramas de flujo de datos correspondiente al sistema de asignación de costos basado en actividades enfocado a la pequeña y mediana industria del sector calzado.

\section{Modelo conceptual del sistema ABC para la industria del calzado}

\subsection{Definición de los procesos}

La cadena de valor típica del sector de la pequeña y mediana industria del calzado está conformada por los procesos principales de diseño, compras, producción, ventas y distribución, así como los procesos de apoyo: mantenimiento y administración. A su vez cada proceso representa una abstracción de un conjunto de actividades.

La presente investigación se limita al proceso de producción y su relación con el proceso de diseño. En éste último diseño se determina cómo serán los mo- delos del calzado, sus especificaciones, los servicios indirectos necesarios para cumplir las exigencias del producto y en consecuencia la factibilidad del mismo. Además, se determina el ciclo de vida probable del calzado, su costo estándar, costo objetivo y se establecen los métodos y niveles de producción para su elaboración. Como fases del proceso de diseño del calzado se identificaron las siguientes: diseño del patrón, elaboración del prototipo, preparación de moldes por tallas.

El proceso productivo de cada modelo de calzado se basa en el diseño previo, transformándose las materias primas en el calzado terminado, a través de las actividades que lo conforman, con los recursos disponibles. Las fases del proceso de producción de calzado identificadas, son: planificación, corte, precostura, costura, sellado, preparación de plantilla y tacón, almacenaje de productos semielaborados, ensamblaje, montura, remate y envase.

En consecuencia, las actividades de diseño conllevan a determinar las actividades de producción para cada modelo, estableciendo el grado de complejidad y el procedimiento necesario.

\subsection{Defínición y descripción de las actividades propias de la industria del calzado}

Se definieron las actividades de producción y diseño de manera que permitan determinar el costo de producción de cada línea de producto; ya que su ejecución genera el consumo de la mano de obra humanos y tecnología, necesarios para transformar la materia prima en productos terminados. Posteriormente se 
agruparon y se describieron para cada línea de producto, ya que el costo de las actividades depende de la complejidad de cada modelo de calzado.

En base al análisis de los procesos, a continuación se presenta de manera secuencial y agrupadas por fases, las actividades del proceso de diseño y producción en dos niveles (Actividades y Subactividades que las conforman), con su descripción, entradas y salidas:

El Proceso de Diseño comprende dos (2) tases:

Fase 1: Desarrollo de los patrones de muestra, lo cual permite desarrollar los patrones en cartón, a través de dos subactividades. Con la primera, denominada selección de modelos, se realiza el estudio y selección de los modelos factibles para la producción y venta, lográndose su dibujo. Posteriormente, la elaboración de la camisa y patrones permite obtener las piezas de cartón que conforman el patrón.

Fase 2: Elaboración de los moldes definitivos. Esta actividad se realiza a través de dos subactividades: La elaboración de muestras, a través de la cual se establece y prueba la factibilidad del proceso productivo del modelo y se producen las muestras en calzado terminado para los vendedores. Por último, se elaboran los moldes definitivos para ser utilizados en los cortes manuales; los cortes para máquinas troqueladoras se elaboran en fábricas especializadas.

El Proceso de Producción requiere dos fases:

La Fase 1 comprende dos (2) actividades y el resultado es la materia prima preparada según la nota de producción por cliente: a) Planificación de la produc- ción en la cual se analizan y clasifican los pedidos para su envio a producción a través de las subactividades de emisión, organización y selección de las notas de producción. La salida final es la nota de producción. b) Preparación de la materia prima se realiza con base en la nota de producción. La salida, según la línea de producto, comprende la materia prima: (piel en partes, piel de forro, miñones, láminas de cartón, láminas de cuero, goma espuma) y los componentes varios (tacones, tela contrafuerte y puntera.)

En la Fase 2 se realizan cuatro (4) macroactividades con el fin de obtener como salida final el corte superior listo:

a) Corte en partes, el cual consiste en cortar la materia prima con métodos manuales o máquinas, según el modelo especificado en la nota de producción. Implica como subactividades el corte de pieles y forros, corte de plantillas y suelas, corte de adornos, corte de miñones y correas, otros cortes. Las salidas de esta etapa son las piezas de piel: pala, centro y trabilla, talón, adornos, plantillas, tacón; las piezas de forro (pala, centro, trabilla), talón, miñones; las piezas de plantillas de cartón, de suela y relleno de sandalia.

b) Pre-costura: se preparan los productos provenientes de la macroactividad anterior para la costura, a través de cinco subactividades: el descarnado para rebajar las piezas cortadas; el empate para unir las piezas del forro y por otra parte las plantillas de piel identificándolas con la numeración; el cerrado y asentado uniendo la parte trasera superior del zapato denominada talón; los refuerzos a través de la colocación de refuerzo a las piezas de piel que conforman la parte externa del calzado; por último la colocación de reto- 
ques y adornos si fuere necesario. La salida de la precostura son las piezas de piel (Pala, trabilla, Centro) reforzadas y con adornos.

c) Costura: Se arman las piezas de piel conformando la parte superior del calzado, obteniéndose el corte semiensamblado. Posteriormente se cosen las piezas y se rematan, eliminando los hilos, pieles pega y cualquier otro material sobrante. Para ello se realizan tres subactividades: el preparado para costura, el cosido y el remate. La salida de ésta es el corte rematado.

d) Sellado del número y modelo en los cortes. Comprende una subactividad con el mismo nombre y su resultado es el corte superior listo.

Fase 3: Se preparan los componentes a través de dos actividades, para obtener como salida la plantilla y el tacón listos para montar:

a) Preparado de las plantillas de cartón dándoles forma según el modelo. En ei caso de sandalias es necesario además, forrar la plantilla con piel. b) Preparado de Tacón: Se forran de piel los tacones de plástico según el modelo.

Fase 4: Denominada depósito o almacenamiento temporal: consiste en la preparación de los productos semielaborados; se agrupa para cada nota de pedido, el corte listo, el tacón con piel, las plantillas de cartón, la plantilla de suela, el contrafuerte, la puntera y materiales varios.

Fase 5 Final: En esta tase se requieren 4 actividades, con el fin de lograr el producto terminado y envasado: a) Ensamblaje. Se refuerza la parte delantera y trasera del calzado con la colocación de punteras y contrafuertes respectivamente. El producto final de esta actividad es el corte reforzado. b) Montura en la horma de la plantilla de cartón, el corte superior listo, la suela y el tacón. El resultado final es el calzado completo montado en la horma. Requiere ejecutar cuatro (4) subactividades según el tipo de montura. c) Remate. Representa la actividad que permite revisar y preparar el calzado para su embalaje, a través de dos (2) subactividades; en la primera, denominada limpieza y brillo, se plancha, pule y desmonta el calzado de la horma; la segunda es el remate final, en la que se adhiere definitivamente el tacón, se coloca la plantilla de piel, se endurece y por último se pule nuevamente el calzado. d) Envase del calzado por par; representa la última actividad del proceso y en ella se revisan y ordenan los pares para ser colocados en cajas debidamente identificadas por modelo, color y número.

En cada empresa el proceso puede tener algunas variantes, de modo que se recomienda reconstruir la descripción y reflejarla en un esquema, con el fin de facilitar la información para elaborar un plan de cuentas base y a la vez visualizar la asignación del costo de los recursos a cada subactividad, actividad y fases del proceso productivo. Los pasos y los recursos inherentes a cada actividad, pueden variar de una empresa a otra, así como los costos. Por lo tanto, se establecerán a la hora de describir las actividades de una empresa en particular, sin afectar las bases propuestas.

\section{3. Formulación del plan de cuentas}

Sobre la base de las actividades definidas anteriormente y de los recursos consumidos (materia prima, recursos humanos y gastos generales) durante los 
procesos típicos del sector, en el Gráfico 1 se presenta una clasificación de cuentas por niveles, general y flexible, de manera que cada empresa la adapte a sus necesidades de información. Así mismo, a continuación se explican los conceptos que conforman el plan:

Recursos Consumidos: (por asignar a las actividades) representan los re- cursos necesarios para la elaboración del calzado terminado. Amerio et al (1997) exponen que la estructura de costos de producción normalmente está conformada por un $46,5 \%$ de Mano de Obra, $48,4 \%$ de Materia Prima y el 5,3\% corresponde a Otros Gastos de Fabricación. Sus consumos en unidades monetarias se asignan a las diferentes actividades que confor-

Gráfico 1

Clasificación de Cuentas por Niveles del Proceso de Producción

\begin{tabular}{|c|c|c|c|}
\hline \multicolumn{2}{|c|}{ 5.0. 00.00 .000} & \multicolumn{2}{|c|}{ Proceso de producción } \\
\hline Clase & Grupo & Cuenta & Auxiliar \\
\hline 5.0 & 5.0 .00 & 5.0 .00 .00 & 5.0 .00 .00 .000 \\
\hline \multirow[t]{3}{*}{$\begin{array}{c}5.1 . \\
\text { Costo de Recursos }\end{array}$} & $\begin{array}{c}5.1 .01 \\
\text { Materia Prima } \\
\quad(\mathrm{MP})\end{array}$ & $\begin{array}{l}\text { 5.1.01.01 Principal } \\
5.1 .01 .02 \text { Materiales varios } \\
\text { 5.1.01.03 M.P.asignada (CR) }\end{array}$ & $\begin{array}{l}\text { Tipo de M.P. } \\
001 \text { Pieles } \\
002 \text { Plantillas } \\
00 \mathrm{n}\end{array}$ \\
\hline & $\begin{array}{c}5.1 .02 \\
\text { Recursos } \\
\text { Humanos } \\
(\mathrm{RH}) \\
\end{array}$ & $\begin{array}{l}\text { 5.1.02.01 Directos } \\
5.1 .02 .02 \text { Indirectos } \\
5.1 .02 .03 \text { R.H. Asignados (CR) }\end{array}$ & $\begin{array}{l}\text { Tipo de R:H: } \\
\text { 001Diseñador } \\
\text { 002Cortadores } \\
\text { 00n }\end{array}$ \\
\hline & $\begin{array}{c}5.1 .03 \\
\text { Gastos } \\
\text { Generales }\end{array}$ & $\begin{array}{l}\text { 5.1.03.01 Suministros } \\
5.1 .03 .02 \text { Depreciaciones } \\
5.1 .03 .03 \text { Mantenimiento } \\
\text { 5.1.03.04 Servicios Regulares } \\
\text { 5.1.03.05 Gastos del personal } \\
5.1 .03 .06 \text { Otros Gastos }\end{array}$ & Detalle del Gasto. \\
\hline $\begin{array}{c}5.2 \\
\text { Costo de actividades }\end{array}$ & $\begin{array}{c}5.2 .01 \\
\text { Costos de } \\
\text { producción }\end{array}$ & $\begin{array}{l}\text { 5.2.01.01 Lanzamiento } \\
\text { 5.2.01.02 Precostura } \\
\text { 5.2.01.0n Demás actividades }\end{array}$ & $\begin{array}{l}\text { Sub actividades } \\
\text { de cada actividad. }\end{array}$ \\
\hline \multirow[t]{2}{*}{$\begin{array}{c}5.3 \\
\text { Costo de los productos }\end{array}$} & $\begin{array}{c}5.3 .01 \\
\text { Productos } \\
\text { en proceso } \\
\text { (PP) } \\
\end{array}$ & $\begin{array}{l}\text { 5.3.01.01 PP } 001 \\
\text { 5.3.01.02 PP 002 } \\
\text { 5.3.01.0n }\end{array}$ & $\begin{array}{l}001 \mathrm{MP} \\
002 \text { Actividad }\end{array}$ \\
\hline & $\begin{array}{l}5.3 .02 \\
\text { Productos } \\
\text { Terminados } \\
\text { (PT) }\end{array}$ & $\begin{array}{l}\text { 5.3.02.01 PT Línea } 1 \\
\text { 5.3.02.01 PT Línea } 2\end{array}$ & \\
\hline
\end{tabular}

Fuente: Elaborado por el Investigador. 
man los procesos y a las entradas y salidas de cada actividad.

Materia prima: se identifica directamente con la producción y está conformada por los componentes básicos del calzado y los materiales necesarios. El costo se mide en términos totales y unitarios según la unidad de medida. Para su cálculo se considera el precio facturado, el transporte, los impuestos aduanales, comisión aduanal y todos aquellos gastos necesarios para que la materia prima arribe al almacén; se expresa en valores unitarios y totales. Se divide en tres cuentas: Materia prima principal, materiales varios y materia prima asignada. A su vez, éstas se deben dividir en los componentes individuales, como piel de corte, de forro, plantillas, suelas, tacón, etc, porque cada componente tiene unidades de medida diferentes. En tal sentido es conveniente clasificarlos y crear una base de datos que contenga el tipo de componente y la unidad de medida para cada uno, lo cual facilitaría el cálculo de los costos por par de calzado. Por ejemplo, la piel se mide por pies, las suelas en gramos y el tacón por unidad.

Recursos Humanos: El costo de los recursos humanos, comprende las remuneraciones y gastos de los trabajadores que laboran en los diferentes procesos. Se compone por el salario fijo más los conceptos que comprenden las Leyes Sociales: prestaciones, vacaciones, utilidades, contribuciones sociales y otros gastos. Al respecto, las cuotas y comisiones representan el salario variable y se miden en función de las unidades producidas. El salario básico, en cambio, representa el salario fijo del empleado y se mide en función de las horas trabajadas.
Por su parte, las leyes sociales afectan porcentualmente, en función de las leyes y el contrato colectivo, tanto al salario fijo como al salario variable.

Gastos Generales: También son comunes a todas las pequeñas y medianas industrias del calzado; por lo tanto, la agrupación por cuentas generales y su descripción puede estandarizarse, permitiendo que cada empresa en particular pueda detallarla según sus objetivos, complementándola con su descripción.

Es importante considerar la incorporación de los efectos de la inflación en todas las cuentas mencionadas; calculados a través de los índices establecidos mensualmente por el Banco Central de Venezuela.

Costo de las Actividades: Representa la segunda clase del plan de cuentas y su división en grupos refleja el costo los procesos que componen la cadena de valor de la empresa. El presente trabajo desarrolla los grupos del costo de producción y diseño, que a su vez se dividen en las cuentas de actividades y subactividades explicadas anteriormente.

Costo de producción por línea. $\mathrm{Ae}$ presenta el costo de las entradas y salidas de cada actividad denominadas productos en proceso, separadas en costo de la materia prima y costo de la actividad (gastos generales + Recursos humanos asignados), hasta llegar al costo de los productos terminados por línea.

El costo de los productos terminados incluye la sumatoria de los costos de las actividades del proceso de producción y el costo de la materia prima consumida por cada unidad. 


\subsection{Determinación de la unidad de trabajo}

Se considera como unidad de trabajo razonable, las horas trabajadas. En ese sentido el costo unitario de cada actividad se calculará dividiendo su costo total durante un período de operación por las horas trabajadas en ese mismo período.

\subsection{Base de asignación de los costos a las actividades}

La distribución de los gastos generales y recursos humanos a las actividades, se realiza de manera porcentual en función de su consumo, como se presenta en el Gráfico 2. A fin de facilitar el trabajo, para la distribución porcentual de los conceptos de costos entre las actividades, se sugieren 2 pasos:

a) Crear un patrón general de distribución porcentual para todos los gastos generales y recursos humanos entre las actividades, a través de una tabla de relaciones. La tabla debe contener de manera horizontal todos los gastos de la empresa o el cargo de los trabajadores (en el caso de la distribución de la nómina) y verticalmente las actividades agrupadas por proceso; por cuanto algunos gastos son absorbidos a la vez por las actividades de producción y las generales. Luego debe establecerse la asignación en términos porcentuales: gasto general/actividad o recurso humano/actividad.

b) Establecer las bases de distribución, ya que varían en función de la naturaleza del gasto, de acuerdo a los siguientes parámetros: En los Suministros de Operación, la base pudiera ser la cantidad de máquinas o herramientas utilizadas en cada actividad. Las depreciaciones y el mantenimiento de los activos fijos se pueden distribuir en función al porcentaje que utiliza cada actividad, hasta un $100 \%$ en el caso de los activos fijos directos. Los gastos por servicios regulares, como por ejemplo la electricidad, el agua, alquileres, entre otros, se pueden distribuir en proporción al consumo por cada actividad. El costo de los recursos humanos puede repartirse estableciendo porcentajes estándar de distribución, basa-

\section{Gráfico 2}

Distribución de los Gastos Generales y los Recursos Humanos

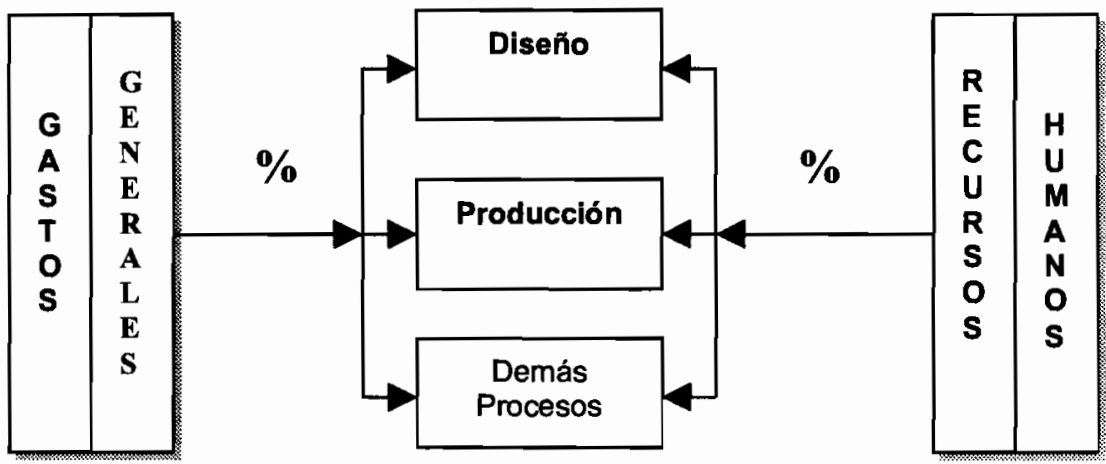

Fuente: Elaborado por el Investigador. 
dos en el tiempo aproximado que ocupan los trabajadores en cada actividad. En tal sentido, se consideran trabajadores directos aquellos que se dedican a una sola actividad $e$ indirectos los que se dedican a varias actividades.

\subsection{Asignación de los costos a las unidades producidas por línea de producto}

La asignación de los costos a los productos se realiza en tres etapas. En la primera etapa se asigna el costo de la materia prima utilizada a cada unidad. Para ello se establecen estándares de las cantidades promedio utilizadas en cada línea de producto, a través de una escala que refleje los intervalos de consumo y permita ubicar cada modelo en una línea determinada. De esta manera se logra una repartición confiable entre los pares producidos.

En la segunda etapa, se calcula el costo total de producción sumando el costo total de la materia prima y el costo de las actividades de producción por línea de producto. Posteriormente, se determina el costo unitario dividiendo el costo total de producción entre los pares fabricados de cada línea. La distribución del costo de las actividades de producción, debe realizarse en función de las horas trabajadas como unidad de trabajo establecida. Es por ello, que, a partir de

\section{Gráfico 3}

Flujograma del Proceso de Asignación de Costos

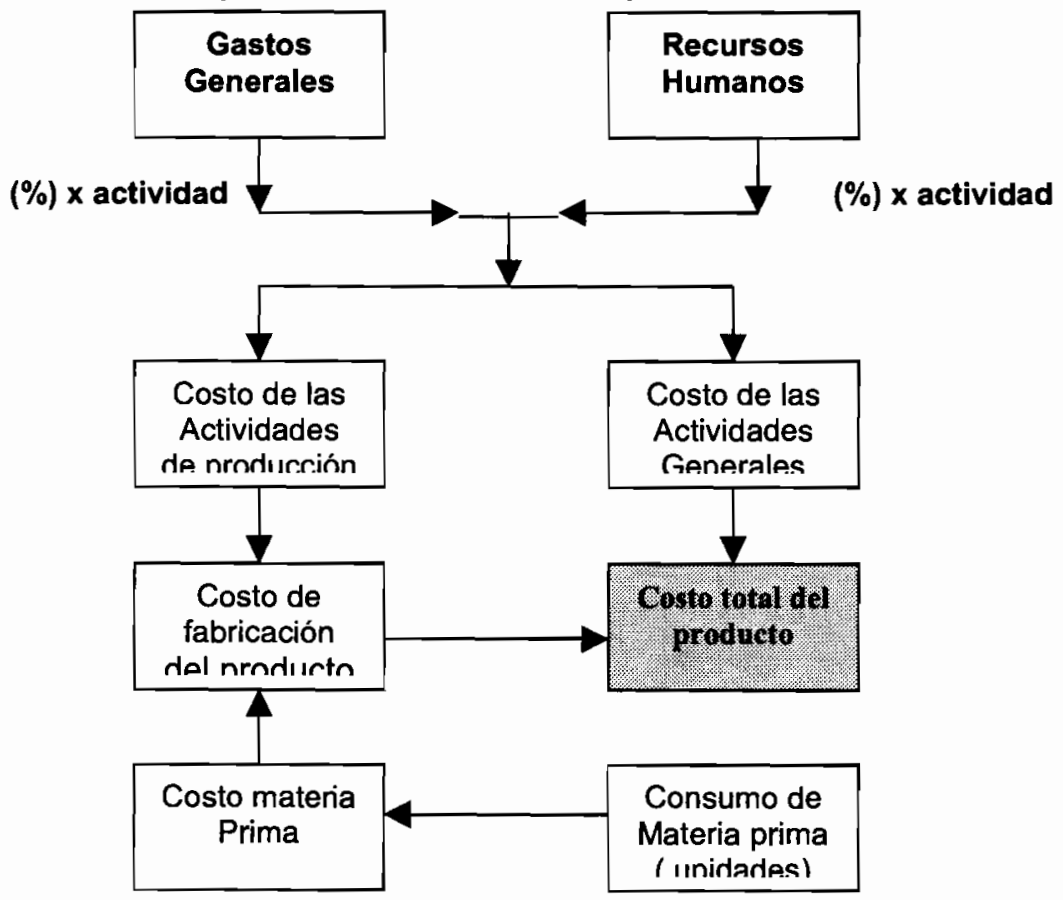

Fuente: Elaborado por el Investigador. 


\section{Gráfico 4}

Diagrama de Flujo de Datos. Nivel $\varnothing$ (Diagrama de Contexto)

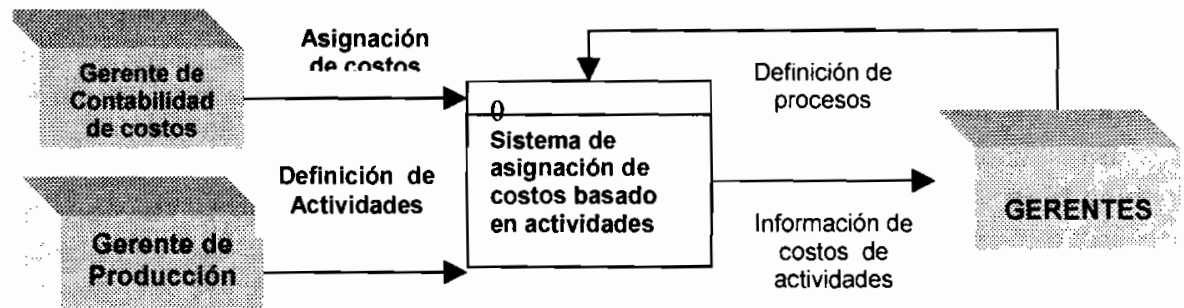

Fuente: Elaborado por el Investigador.

un estudio de tiempo, se establecen las horas promedio necesarias para realizar cada actividad por línea de producto. La información se mantiene en una base de datos. Cada vez que surjan nuevas líneas y a medida que se mejoren los procesos productivos, es recomendable la creación o modificación de la base de datos del proceso, actividad y línea de producto; así como la revisión y modificación de las tablas de estándares que resumen la información.

Por último, en una tercera etapa se puede determinar el costo total, agregando al costo total de producción de un período, el costo de las actividades que conforman los demás procesos de la cadena de valor, del mismo período. La asignación del costo de los demás procesos a cada línea de producto se realiza en proporción al costo de producción de cada una. El costo total por unidad producida se calcula dividiendo el costo total por línea entre las unidades producidas de cada línea.

A manera de resumen final, el proceso para introducir las bases de asignación de costos se presenta en el Gráfico 3 y representa un plan general que cubre el largo plazo. Por tal motivo deben ser revisadas periódicamente con la finalidad de actualizar y mejorar continuamente el sistema, según las exigencias del entorno. Por lo tanto los gerentes de costos que lo incorporen, deben ser reactivos y proactivos.

En el diagrama de Flujo de Datos presentado en el Gráfico 4, se visualiza globalmente el proceso anterior.

\section{Conclusiones y recomendaciones}

Las bases conceptuales de los sistemas de costos deben adaptarse a la estructura de costos y a los procesos productivos de cada sector industrial en particular. Además deben relacionarse con las teorías modernas utilizadas en el ámbito internacional, tanto en el área contable como gerencial.

El sistema de asignación de costos basado en actividades propuesto, sine de soporte para que los sistemas administrativos y contables suministren a los gerentes información de costos relacionada con los procesos productivos, las actividades que los conforman y los productos. Por lo tanto, su análisis e interpre- 
Sistema de costos. Sector Calzado Amerio, María Anna et al.

tación permitirá gerenciar por actividades y alcanzar el mejoramiento continuo.

En consecuencia las decisiones se orientarán a lograr incrementos en la calidad, productividad y por ende en la competitividad.

Una vez implantado el sistema, es recomendabie extenderlo a los demás procesos que conforman la cadena de valor de la industria.

Además, es importante considerar y poner en práctica sistemas de presupuestos y contabilidad automatizados con el fin de procesar de manera planificada y organizada la información de costos, estimada y real, para suministrar los reportes justo a tiempo, de acuerdo con los requerimientos gerenciales.

\section{Bibliografía citada}

Acosta, F.(1996). Costo Basado en Actividades, Congreso de la Federación de Contadores Públicos de Venezuela, 22-11-1996.

Amerio, M.; Viloria, O. y Nava, L. (1997). La contabilidad de costos en la pequeña y mediana industria venezolana del calzado, Revista Venezolana de Gerencia, Año 2, № 3, Junio 1997. p. 67-76.

Andersen, A. (1996). El Costeo Basado en Actividades, Revista Reinventando el Costo, Venezuela Vol. 1 p. 4-9.

Baker, W. (1994). Understanding Activity Based Costing, Review Finance, USA, March, April, p. 28-30.

Burgos, I. (1994). Decisión ¿Laberinto Gerencial?, UCV, 165 p. Venezuela.
Castillo, F. (1995). El sistema de costos ABC, La Contabilidad de gestión en los 90: 50 artículos divulgativos, AECA, España, p. 257-260.

Enright, M.; Francés, A. y Saavedra, E. (1994). Venezuela: el reto de la competitividad, Ediciones IESA, p. 617-656. Venezuela.

FIM Productividad, (1992). Mejoramiento Continuo de Calidad y Productividad, Editorial Fim, 96 p. Venezuela.

Horngren, Ch.; Foster,G. y Srikant, M. (1996). Contabilidad de Costos un enfoque gerencial, Editorial Prentice Hall, 970 p. México.

Howell, Ry Soucy, S. (1991). Determining The Real Costs of Doing Business in a Global Market, National Productivity Review, p.57-165.

Kaplan, R. (1994). Llega la Revolución Contable. Revista Entre Gerentes. № 597, Abril. p. 51.

Kendall, K. y Kendall, J. (1997). Análisis y Diseño de Sistemas, Editorial Prentice Hall, p. 229-274, México.

Linares, R. (1996). Estrategias gerenciales para la pequeña y mediana empresa, Ediciones IESA, 124 p., Venezuela.

Lorino, P. (1993). El control de gestión estratégico, Ediciones Marcombo S.A., 193 p., Barcelona.

Polimeni, R., Fabozzi, F. y Adelberg, A.(1994). Contabilidad de Costos Conceptos y Aplicaciones para la Toma de Decisjones Gerenciales, Editorial Nomos S.A., 780 p. Colombia.

Shank, J. y Govindarajan, V. (1996). Gerencia Estratégica de Costos, Editoriai Norma S.A., 340 p., Colombia. 